\title{
Influence of classical resonances on chaotic tunnelling
}

\author{
Amaury Mouchet \\ ${ }^{1}$ Laboratoire de Mathématiques et de Physique Théorique (CNRS UMR 6083), \\ Université François Rabelais Avenue Monge, Parc de Grandmont 37200 Tours, France \\ Christopher Eltschka and Peter Schlagheck \\ Institut für Theoretische Physik, Universität Regensburg, 93040 Regensburg, Germany
}

(Dated: May 3, 2019)

\begin{abstract}
Dynamical tunnelling between symmetry-related stable modes is studied in the periodically driven pendulum. We present strong evidence that the tunnelling process is governed by nonlinear resonances that manifest within the regular phase-space islands on which the stable modes are localized. By means of a quantitative numerical study of the corresponding Floquet problem, we identify the trace of such resonances not only in the level splittings between near-degenerate quantum states, where they lead to prominent plateau structures, but also in overlap matrix elements of the Floquet eigenstates, which reveal characteristic sequences of avoided crossings in the Floquet spectrum. The semiclassical theory of resonance-assisted tunnelling yields good overall agreement with the quantum-tunnelling rates, and indicates that partial barriers within the chaos might play a prominent role.

PACS numbers: $05.45 . \mathrm{Mt}, 05.60 . \mathrm{Gg}, 32.80 . \mathrm{Qk}, 05.45 . \mathrm{Pq}$,
\end{abstract}

\section{INTRODUCTION}

Since the very first quantitative studies of tunnelling in a chaotic system [1, 2], it has been clear that a minute scrutiny of the associated classical dynamics was required in order to understand even the most coarse features of the quantum behaviour of such systems. In essence, tunnelling is a semiclassical concept since it refers to a quantum process - typically a decay or the oscillation of an averaged observable - that is forbidden at a classical level. But, despite the numerous successes of semiclassical computations in quantum chaos, the questions of which and how classical objects can be used to understand tunnelling and to compute, say, their characteristic time scales, have been remaining widely open for sixteen years. The stakes in the battle are important since non-integrability is the generic rule of multidimensional systems and tunnelling may play a crucial rôle in their transport properties. Moreover, it is one of its signatures that chaotic tunnelling can be modified on several orders of magnitude by the slightest variation of any classical or quantum parameter; therefore a deep understanding of chaotic tunnelling is required to control the process, what may be an advantage in delicate quantum experiments and, hopefully, give rise to an extremely sensitive quantum tool. Some promising clues have been provided in this direction by numerical studies and experiments with cold atoms [3, 4, 5, [6] but also with microwave cavities [7, 8] where tunnelling signatures for processes that are forbidden by ray optics were observed.

Of course, in order to capture the typical exponentially

*email: mouchet@phys.univ-tours.fr

† email: Peter.Schlagheck@physik.uni-regensburg.de small tunnelling effects, it is expected that the classical dynamics should be complexified. It is well-known [9, 10] that the complex solutions of Hamilton's equations are actually involved in the interpretation of tunnelling of autonomous systems with one degree of freedom. In the mid 90's, the first observations 11, 12, 13, 14] that complex periodic orbits allow one to reproduce quantitatively some feature of chaotic tunnelling gave hope that a semiclassical strategy was indeed possible, even though the complexified classical tori are generically destroyed in chaotic systems [15]. But to deal with tractable semiclassical trace formulae à la Gutzwiller, a general criterion for selecting the complex periodic orbits was still lacking; this need became an emergency when it was unexpectedly discovered [16, 17] that chaos reveals itself in the complex phase-space through some fractal structures, the so-called Laputa islands, that look like agglomerates of complex trajectories. It is only recently that some encouraging significant steps were done for retaining the relevant semiclassical skeleton 18. A lot of work remains to be done in that direction, especially if one wants to deal with continuous systems where time can (and must) be complexified as well, unlike what occurs in discrete maps.

The second strategy to cope with chaotic tunnelling is not purely semiclassical but rather calls up random matrix theory. Since the seminal work presented in Ref. [19], a fruitful approach of quantum chaos is to replace a chaotic but deterministic Hamiltonian by a random element of an ensemble of matrices that only encapsulates the global symmetries. These hybrid techniques, with both semiclassical and statistical ingredients, first allowed us to qualitatively understand the so-called chaosassisted tunnelling, i.e the observation [20] that tunnelling is increased on average as the transport through chaotic regions grows [21, 22, 23]. However, the extreme 
sensitivity of tunnelling renders the predictions very difficult even if just an order of magnitude is required. This is also true for the seemingly simple case of nearintegrable dynamics where it was shown, on a discrete quasi-integrable quantum map, that the internal resonances may enhance the transitions by several orders of magnitudes [24, 25]. This resonance-assisted tunnelling is also at work in discrete systems where chaos is much more developed [26]. The aim of the present paper is to show that the ideas in Refs. 26, 27] are strengthened, now in a continuous system, by a systematic analysis of level dynamics and the phase-space representation of the quantum states.

We shall begin in section II with a short presentation of the general framework of chaotic tunnelling and the model we choose in order to study it. We will be concerned with a typical signature of tunnelling, namely the period of Rabi oscillations between two wells that are separated by a dynamical barrier. Then we will show (section IIII) with a simple argument, that the attempt to reproduce the average tunnelling periods presented in Ref. [28] is far from being complete precisely because it ignores the resonances, among other things. In sectionIV we will give an illuminating illustration of a characteristic feature of chaotic tunnelling [29] : it appears to be a collective effect in level dynamics where not just one third state crosses a tunnelling doublet. We will give a phase-space picture of resonance-assisted tunnelling and confirm, that taking into account the resonances is unavoidable if we want to reproduce or predict the average behaviour of tunnelling transitions. In section $\nabla$ we show that the ideas of Refs. 26, 27] actually provide a good estimate for the average tunnelling rate in our model.

\section{GENERAL FRAMEWORK OF CHAOTIC TUNNELLING}

The simplest non-integrable Hamiltonian models are either time-dependent one-dimensional (1D) systems or, equivalently, autonomous systems with two degrees of freedom where the Hamiltonian is the only constant of motion. Seen from the classical point of view, a generic potential induces a cascade of non-linear resonances whose overlap generates chaos [30, 31]. One minimal continuous model that encapsulates these typical properties is a 1D time-dependent system whose Hamiltonian is

$H(p, q ; t)=\frac{p^{2}}{2}-\frac{\gamma_{+}}{2} \cos (q+2 \pi t / \tau)-\frac{\gamma_{-}}{2} \cos (q-2 \pi t / \tau)$,

where $p$ and $q$ denote canonical action-angle variables that are coordinates on a phase-space having the topology of a cylinder: it is $2 \pi$-periodic in position (angle) $q$ and infinitely extended in the momentum (action) direction. The model (1) can be seen as the most natural normal form where we keep only two overlapping reso- nances i.e. the first two time-dependent Fourier components of the potential. The Hamiltonian $H$ can also be interpreted, provided a change of frame is performed, as a pendulum driven by a periodic wave. Moreover, it is actually very similar to the effective Hamiltonian that can be realised in experiments on cold atoms [32]. We shall consider the period $\tau \equiv 2 \pi$ in the following.

The two parameters $\gamma_{\mp}$ control the size of the two stable islands $\mathcal{I}_{ \pm}$located in phase-space near $p= \pm 1$ respectively. When increasing the $\gamma$ 's from zero, we leave the quasi-integrable free-like motion and rapidly (at $\gamma \sim 0.2$ ) reach a mixed regime where the two stable resonance islands are fully surrounded by a chaotic sea. If an initial condition lies inside one island, the classical motion will remain trapped forever within a very thin quasi-one dimensional layer without the possibility of escape (this evasion is the forbidden process that tunnelling will be concerned with $[33 \mid$ ). Alternatively, a trajectory starting in between the two islands is chaotic: without being fully ergodic since it cannot enter the islands, the absence of a sufficiently large number of constants of motion allows the system to explore large areas and to develop an exponential sensitivity on initial conditions. Still increasing the $\gamma$ 's, the cascade of secondary resonances inside the islands erode gradually the regular zones and make them dissolve completely in the chaotic sea. Figure 1 shows some Poincaré sections of the classical dynamics, i.e views taken stroboscopically at every integer multiple of the time period $\tau=2 \pi$ of (11).

At a quantum level, the quantization of a Hamiltonian like (10) has, for a long time, been employed as a very natural continuous model for studying tunnelling [34, 35, 36]. The time-dependence is implemented within the Floquet theory where the phases $\epsilon$ of the eigenvalues of the evolution operator at time $\tau$ play the rôle of the energies for autonomous systems. The eigenvectors associated with these so-called quasi-energies $\epsilon$ will be called the (eigen)states of the system. When $\hbar$ is small enough compared to the typical size of the stable islands, some states, the regular states $\left\{\left|\phi_{n}\right\rangle\right\}_{n \in\{0,1, \ldots\}}$, appear to have their Husimi distribution localised on the two main stable islands while other states, the chaotic states, are found to be delocalised in the chaotic sea.

Following the same route that is explained in great detail in Ref. [29, $\S \S$ II \& III], we will keep a discrete two-fold symmetry, namely the time-reversal symmetry, by considering $\gamma_{+}=\gamma_{-}=\gamma$ throughout this paper [37]. This allows us to clearly identify the tunnelling process through the existence of small energy scales, the doublet splittings $\Delta \epsilon_{n}$, which are associated with large time scales, namely the periods $2 \pi \hbar / \Delta \epsilon_{n} \gg \tau$ of tunnelling oscillations between the stable islands. In other words, the magnitude of the splittings $\Delta \epsilon_{n}=\left|\epsilon_{n}^{+}-\epsilon_{n}^{-}\right|$between the states that are symmetric $\left(\left|\phi_{n}^{+}\right\rangle\right)$and antisymmetric $\left(\left|\phi_{n}^{-}\right\rangle\right)$with respect to the time-reversal symmetry measure the importance of tunnelling between the two stable islands. The actual challenge of chaotic tunnelling is to understand and hopefully predict the behaviour of $\Delta \epsilon_{n}$ as 


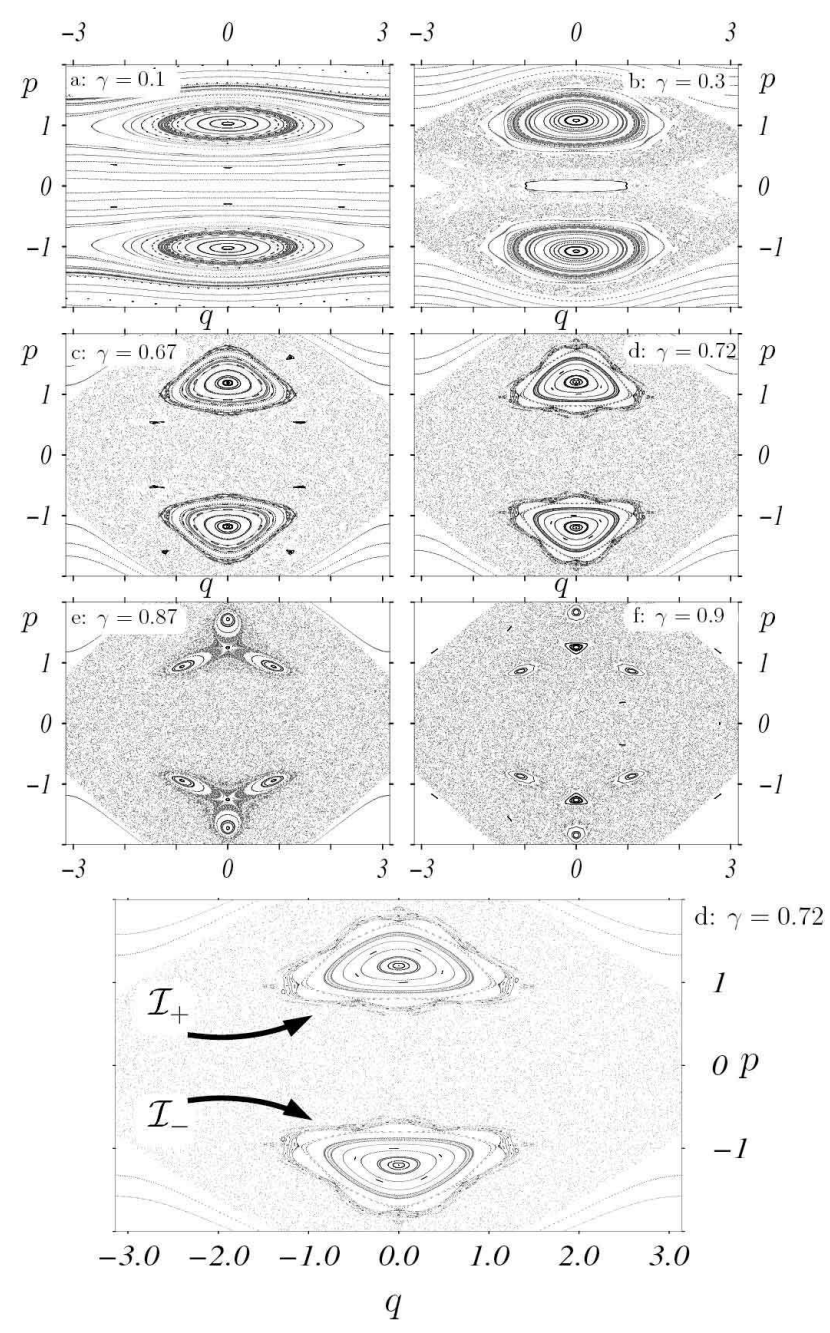

FIG. 1: Some Poincaré sections ( $\tau=2 \pi$-stroboscopic plots) corresponding to Hamiltonian (1) for several values of $\gamma_{+}=$ $\gamma_{-}=\gamma$. Increasing $\gamma$, between $\gamma \simeq 0.1$ and $\gamma \simeq 0.3$, the two resonances at $(p, q) \simeq( \pm 1,0)$ start to significantly overlap, and the systems enter in a mixed regime where both regular and chaotic dynamics coexist. From $\gamma \sim 0.3$ to $\gamma \sim 0.8$, the two stable islands $\mathcal{I}_{ \pm}$are completely surrounded by a chaotic sea, and although they are related by the timereversal symmetry, they are disconnected from each other at a classical level, since dynamical barriers prevent the real classical trajectories starting in one island to escape from it. For $\gamma \sim 0.72, \mathcal{I}_{ \pm}$have developed a relatively wide $3 / 7$ resonance, which is not the case for $\gamma \sim 0.67$ where the corresponding $3 / 7$ resonance chain is hardly visible. The insets in FIG. 2] show magnifications of the islands in these last two cases.

a classical $(\gamma)$ or quantum $(\hbar)$ parameter is varied within a regime where the quantum scales are small enough to resolve the classical scales of the chaotic structures.

Unlike what occurs for integrable multidimensional systems where $\Delta \epsilon_{n}$ is a smooth monotonic function given by $\Delta \epsilon \propto \mathrm{e}^{-A / \hbar} \quad 38$ with $A$ being a typical classical action that characterizes the tunnelling barriers, the split- tings can display huge fluctuations in the mixed regularchaotic case, which were soon identified as a signature of chaos [22, 34]. But as far as isolated fluctuations are observed this point of view must be amended. One isolated fluctuation is clearly associated with a third state whose (quasi)energy nearly degenerates with the doublet [34, 36] as $\gamma$ or $\hbar$ is varied. Chaos is not necessarily relevant here since such fluctuations can be observed when the chaotic layers are too small (and $\hbar$ too large) to be resolved by the quantum waves. It may happen that the third state is also a regular state localised on another regular EBK torus, possibly belonging to another stable island. What deserves the name of chaotic tunnelling is a radical change of regime where the fluctuations are not isolated anymore and where the coarse-grained behaviour of $\Delta \epsilon$ does not follow a monotonic law. It was shown in Ref. [29, § V], that there actually exists a rather abrupt transition between a quasi-integrable tunnelling regime and a chaotic regime and that this transition occurs precisely when the quantum eyes can resolve the chaotic classical structures.

While the study of statistics of the splittings is necessary to give an insight into chaotic tunnelling [23, 39], we will focus here on the average behaviour of the splittings. It is far from obvious that we can conceptually justify the distinction between "large" scales (the average) and "small" scales (the fluctuations) in the variations of $\Delta \epsilon$. Most probably, there may exist a whole hierarchy of such variations. From the numerical point of view, however, there seems to be an overall modulation of $\Delta \epsilon$, both observed in maps (40, FIG. 3], 26, FIGs. 1 \& 2]) and in continuous systems [3, FIG. 7]. This modulation is precisely the very object of Ref. [26] (see also Ref. [27]) and of the present paper.

\section{KEY RÔLE OF RESONANCES}

In the general context recalled in the previous section, where tunnelling transitions occur between two symmetric but disconnected stable classical islands, the following estimate has been proposed for a typical chaotic tunnelling splitting [28, eq. (4)]:

$$
\Delta \epsilon \simeq \hbar \Omega \frac{\Gamma(2 N, 4 N)}{\Gamma(2 N+1 ; 0)} .
$$

Here, $\Gamma$ stands for the incomplete Gamma function, $N=A /(2 \pi \hbar)$ denotes the semiclassical estimate of the number of states localised in one island of area $A$, and $\Omega$ represents an unknown prefactor, with the dimension of an inverse time scale, which does not depend on $\hbar$. Though the origin of the formula remains obscure as Eq. (2) is not explicitly proven by their authors, it gave, in Ref. 28], good agreement with numerical computations provided we are ready to accept an unreasonably large ambiguity on the unspecified proportionality factor: for $\hbar^{-1} \simeq 40$, the estimate (2) varies by five orders 
of magnitude $\left(10^{-1}\right.$ versus $\left.10^{-6}\right)$ in the two cases considered in FIG. 2 of Ref. [28] where the areas $A$ of the stable islands are of the same order. More recent calculations within the "kicked Harper" model, however, revealed substantial deviations between Eq. (2) and the exact quantum tunnelling rates in the semiclassical regime, where a reasonable agreement was only found in the deep quantum limit of large $\hbar$ [27].

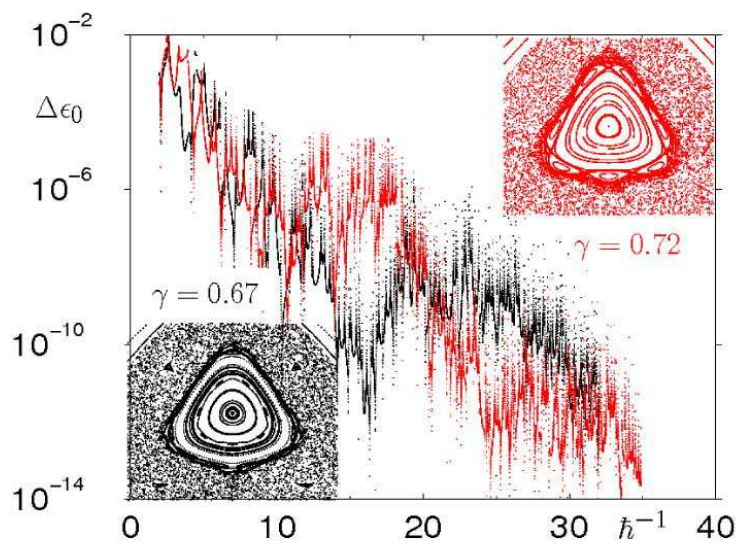

FIG. 2: (Color online) Comparison of the tunnelling splittings $\Delta \epsilon_{0}$ for $\gamma=0.72$ (black line) and $\gamma=0.67$ (gray $/$ red line). The corresponding stable islands, magnified in both cases with the same scaling factor, are shown in the insets (see FIG. 1 c) and d) for a full Poincaré surface of section). The exponentially large difference between the two curves is explained by the different internal structure of the islands: for $\gamma=0.72$, the $3 / 7$ resonance chain is much more developed than for $\gamma=0.67$.

Indeed, no good estimate of $\Delta \epsilon$ can be obtained if the only classical parameter on which the theory depends is the area $A$ of the island. As shown in FIG. 2] the internal classical structure of the islands must be involved in one way or another. We have plotted here the splitting $\Delta \epsilon_{0}$ between the two "central" states $\left|\phi_{0}^{ \pm}\right\rangle$localised in the two islands; more precisely, they both were selected by the criterion of having the maximal overlap with a coherent state that is located on the central stable periodic orbit of period $\tau$. The two graphs, $\Delta \epsilon_{0}$ as a function of $1 / \hbar$, are shown for the two classical parameters $\gamma=0.67$ and $\gamma=0.72$ (see also FIG 1 c) and d)). In these cases, the stable symmetric islands $\mathcal{I}_{ \pm}$ have an area $A$ of the same order of magnitude, but exhibit a rather distinct internal structure: for $\gamma=0.72$, $\mathcal{I}_{ \pm}$have developed a wide $3 / 7$-resonance chain compared

Let us fix the classical dynamics at $\gamma=0.72$ where, as we have seen in the previous section, the internal 3/7resonance is suspected to provoke the enhancement of average tunnelling for $11 \lesssim 1 / \hbar \lesssim 19$ (see FIG. 21). When to the $\gamma=0.67$ case. In the log-plot, the two graphs strongly differ. Not only the fluctuations of $\Delta \epsilon_{0}$ hardly match, but also the average behaviour is completely distinct in a semiclassical regime where $1 / \hbar>10$. A discrepancy of about five orders of magnitude can be clearly observed for $1 / \hbar \simeq 16$ : for $\gamma=0.72$, we have $\Delta \epsilon_{0} \sim 10^{-6}$ compared to $\Delta \epsilon_{0} \sim 10^{-11}$ for $\gamma=0.67$. At $1 / \hbar \sim 25$, on the other hand, the splittings for $\gamma=0.72$ are about $10^{-3}$ smaller than for $\gamma=0.67$.

In any case, no estimate formula $\Delta \epsilon_{0}(A, \hbar)$, which would produce approximately the same graphs for $\gamma=$ 0.67 and $\gamma=0.72$, can be satisfactory. At this stage it is more than plausible that the internal structure of $\mathcal{I}_{ \pm}$must be taken into account in any attempt to estimate $\Delta \epsilon_{n}$ with the help of classical ingredients.

\section{MULTILEVEL CROSSING}

To obtain more insight, we analyze in this section the "level dynamics", i.e. the changes of the quasi-energy spectrum as $1 / \hbar$ or $\gamma$ is varied. This can, for instance, explain individual fluctuations spikes, which correspond to the crossing of the tunnelling doublet with a third state (or an unresolved doublet) 22, FIG. 7] [34, FIG. 1], 29, FIG. 4]. As far as the average of $\Delta \epsilon$ is concerned, we can also establish a correspondence between its behaviour as a function of $1 / \hbar$ and some features of the level dynamics. First, a technical point should be mentioned at this stage. For $\tau$-periodic systems whose classical phase-space is unbounded, it is expected that the quasi-energy spectrum becomes a dense set because of the foldings of an infinite spectrum in the finite Floquet zone $]-\pi \hbar / \tau, \pi \hbar / \tau]$. Hence, we need a criterion to select only those states that are relevant in the level dynamics. Numerically the truncation of the Floquet matrices at high $|p|$ 's is not sufficient, especially for small $\hbar$, since more and more regular and chaotic states are a priori potentially implicated in the tunnelling dynamics. To select the levels that are actually involved, we will use a simple criterion based on the systematic computation of the overlaps $\sigma_{m}$ between the states $\left|\psi_{m}\right\rangle$ and a coherent state centered on the island: $\sigma_{m} \stackrel{\text { def }}{=}\left|\left\langle z_{\text {stable orbit }} \mid \psi_{m}\right\rangle\right|$. Retaining only those states whose $\sigma_{m}$ is larger than a given threshold $\sigma_{\text {filter }}$ will filter the levels that are the best candidates to play a rôle in the tunnelling process, precisely because their wave-functions are not negligible in the area where the tunnelling doublet wave-functions live.

we look at this plateau more carefully, we can identify two "bumps" that are visualised in FIG. 3 One corresponds to $11 \lesssim 1 / \hbar \lesssim 13.5$ followed by a wider one

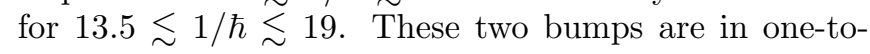



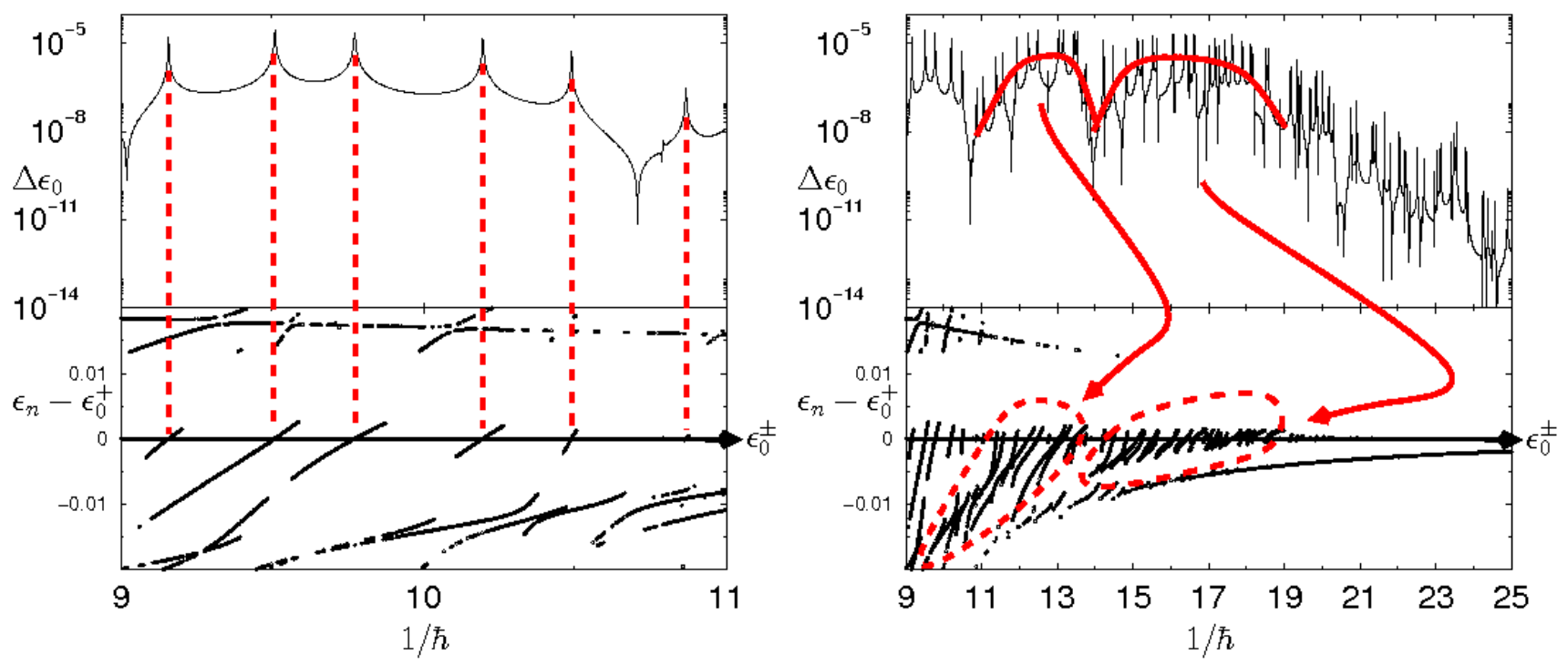

FIG. 3: (Color online) The upper panels show $\Delta \epsilon_{0}$ as a function of $1 / \hbar$ for $\gamma=0.72$ and the lower panels display a part of the corresponding Floquet spectrum. Only the quasi-energies associated with states whose overlaps are $\sigma_{m} \geq 7 \times 10^{-3}$ are shown, once calibrated with respect to the tunnelling doublet $\epsilon_{0}^{ \pm}$(unresolved in the lower panels). For relatively large $\hbar$ (on the left-hand side), the huge fluctuations of $\Delta \epsilon_{0}$ are in one-to-one correspondance with the crossings of the tunnelling doublet by one isolated doublet. On the right-hand side, the upper panel shows a magnification of the plateau in $\Delta \epsilon_{0}$ occurring at $\gamma=0.72$ between $\hbar \simeq 11$ and $\hbar \simeq 19$ (see also FIG. 2). The lower right panel displays a part of the corresponding Floquet spectrum. The plateau in $\Delta \epsilon_{0}$ corresponds to a large number of crossings where we can even identify by eye two families of levels (encircled by the dashed lines) that give rise to the two bumps in the average of $\Delta \epsilon_{0}$ (red/grey thick continuous line in the upper right panel). Beyond the point where the plateau ends, i.e. for $\hbar^{-1} \gtrsim 19$, no more crossings can be identified, and the only other state apart from $\epsilon_{0}^{ \pm}$that significantly overlaps with the Husimi function in the island is the bystander doublet $\epsilon_{5}^{ \pm}$ (see also FIG. 41).

one correspondence with two bunches of levels with significant overlaps $\sigma_{m}$ crossing the tunnelling doublet $\epsilon_{0}^{ \pm}$. More generally, we have observed in many cases, with such a level-dynamics point of view, that there seems to be a clear change of regime: for a given $\sigma_{\text {filter }}$, when the average of $\Delta \epsilon_{n}$ stops decreasing, the states $\left|\psi_{m}\right\rangle$ whose quasi-energies are in the neighbourhood of $\epsilon_{n}^{ \pm}$ and whose $\sigma_{m}$ are larger than $\sigma_{\text {filter }}$ become significantly more numerous. For instance, in FIG. [3] where

In order to be more convincing, this qualitative observation can be strengthened by a phase-space analysis. In addition to the unresolved tunnelling doublet $\epsilon_{ \pm}^{0}$, we can clearly see, in the level dynamics in FIG. [4 two other unresolved doublets $\epsilon_{5}^{ \pm}$and $\epsilon_{7}^{ \pm}$for $1 / \hbar \gtrsim 20$ whose Husimi distribution is mainly located near the boundary of the stable islands $\mathcal{I}_{ \pm}$. In fact, the doublet $\epsilon_{5}^{ \pm}$can be followed along a quasi-continuous line even for $1 / \hbar \leqslant 20$ whereas the other one cannot be identified unambiguously in that region of $\hbar$ corresponding to the plateau: we chose $\sigma_{\text {filter }} \simeq 7 \times 10^{-3}$, or in FIG. 4 with a five times more rough filter $\sigma_{\text {filter }} \simeq 1.4 \times 10^{-3}$, just after the end of the large plateau at $1 / \hbar \gtrsim 20$, the crossings with relevant levels become suddenly scarce. To put it in a different way, the average enhancement of tunnelling appears to be the outcome of a collective dynamics involving numerous states that can be seen in the spectrum through their crossing with the tunnelling doublet.

when $1 / \hbar$ is decreased from about 19 , the doublet line $\epsilon_{7}^{ \pm}$ encounters many avoided crossings and ramifies into the bunch of levels we have precisely associated with the bump. Therefore, even if their Husimi plots both look very similar, one doublet is dramatically involved in the tunnelling process while the other remains a bystander. If we compute the overlap of these states with the harmonic states inside the islands, that is, the eigenstates of the harmonic approximation of the Hamiltonian (1) near the corresponding stable periodic orbits, we find that the by- 


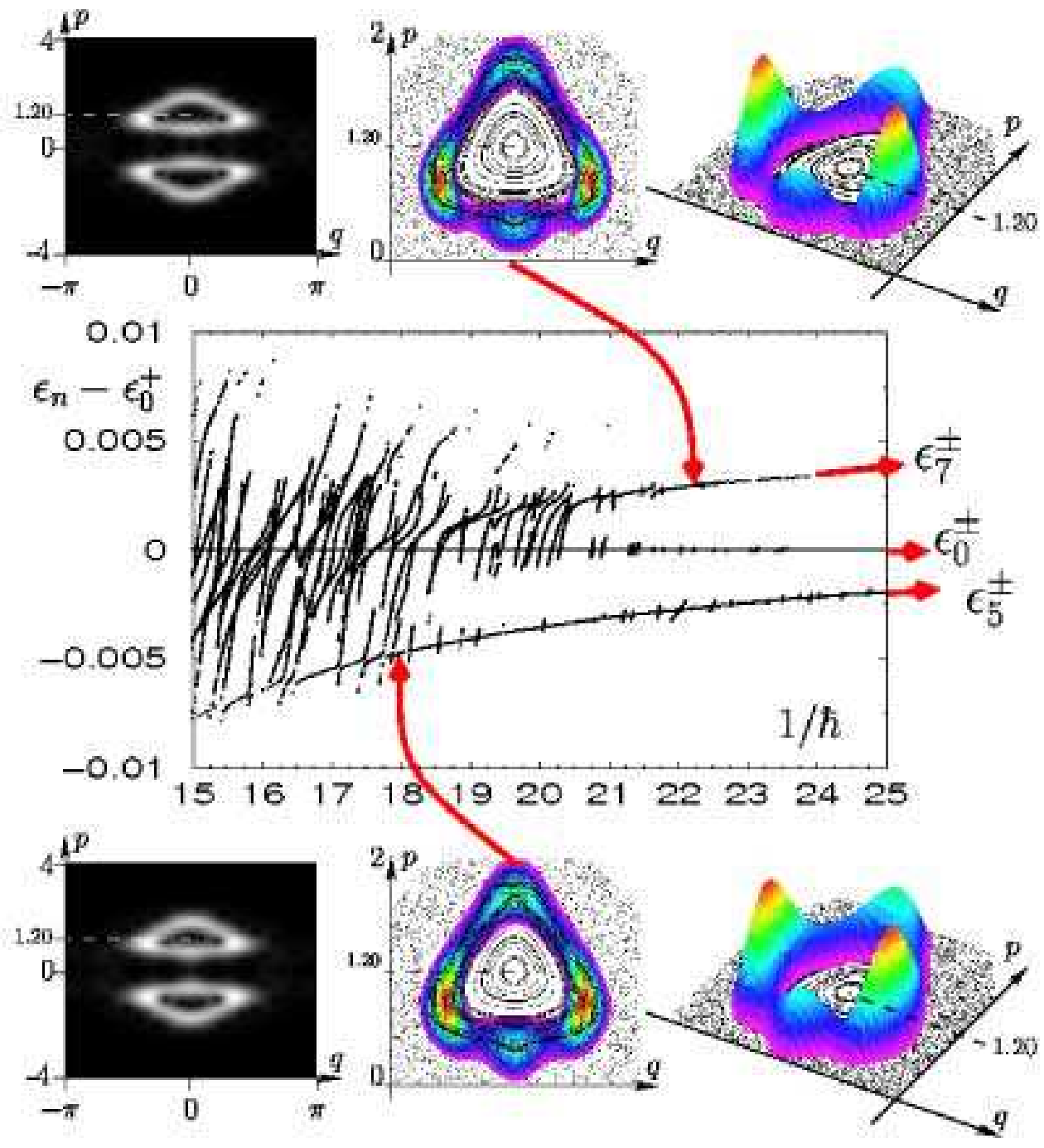

FIG. 4: (Color online) The level dynamics for $\gamma=0.72$ calibrated with respect to the tunnelling doublet $\epsilon_{0}^{ \pm}$(unresolved). Compared to FIG. 3] more states are plotted since we take a smaller $\sigma_{\text {filter }} \simeq 1.4 \times 10^{-3}$. When looking at the crossings with the tunnelling doublet, there still is a clear transition of regime for $\hbar^{-1}$ below or above 20, which corresponds to the end of the plateau in $\Delta \epsilon_{0}$. The three upper views show the Husimi distribution of the state, $\left|\phi_{7}^{+}\right\rangle$, whose quasi-energy is $\epsilon_{7}^{+}$, for $\hbar^{-1}>20$. A comparison with the Poincaré surface of section in the neighbourhood of the stable island $\mathcal{I}_{+}$is shown. The three lower views (which are, actually, very similar to the upper ones at this resolution) correspond to $\left|\phi_{5}^{+}\right\rangle$, the symmetric state whose quasi-energy is $\epsilon_{5}^{+}$can be followed for every $\hbar^{-1}$. Unlike $\left|\phi_{7}^{ \pm}\right\rangle$, the doublet $\left|\phi_{5}^{ \pm}\right\rangle$does not cross and has no influence on the tunnelling doublet. $\left|\phi_{7}^{+}\right\rangle$(resp. $\left|\phi_{5}^{+}\right\rangle$) is localised near the boundary of the two islands and indeed corresponds to the symmetric combination of the 7 th (resp. 5th) excited regular state in $\mathcal{I}_{ \pm}$.

stander doublet $\epsilon_{5}^{ \pm}$is indeed the 5 th excited doublet in $\mathcal{I}_{ \pm}$ whereas the other $\epsilon_{7}^{ \pm}$is the 7 th. This can also be checked in the Floquet spectrum. Indeed, we expect that the levels of the local eigenmodes of the island (which is locally equivalent to a harmonic oscillator) approximately differ from each other by multiples of $\hbar \omega_{0}$ where $\omega_{0} \simeq 0.4$ denotes the frequency oscillations around the center of the island at $(p, q) \simeq( \pm 1.20,0)$. This yields $\epsilon_{l}^{ \pm} \simeq \epsilon_{0}^{ \pm}+l \hbar \omega_{0}$ modulo a Floquet width $2 \pi \hbar / \tau$ for the levels. The fact that it is precisely the quantum number $\ell=7$ which is involved in the emergence of the plateau is not a coincidence. This $\ell$ is exactly the order of the resonance $3 / 7$ that dominates in $\mathcal{I}_{ \pm}$for $\gamma=0.72$.

The quantitative details of how the classical resonances may be implemented in order to reproduce the average behaviour of $\Delta \epsilon_{0}$ will be described in the next section. Even though the approximations that are involved are not always under rigorous control, we can see here the resonances at work. Suppose that the classical parameters are such that one classical resonance $s / \ell$ dominates 
the others in the stable islands $\mathcal{I}_{ \pm}$( $s$ being an integer and $\ell$ a strictly positive integer, the order of the resonance). This precisely means that one torus is actually broken into a chain of $\ell$ sub-islands centered about a stable periodic orbit of period $2 \pi / \omega_{0}=\ell \tau / s$. A quantum resonance occurs when two quasi-energies $\epsilon_{m}$ and $\epsilon_{0}$ are nearly degenerate, i.e. differ by an integer number $s^{\prime}$ of Floquet widths: $\epsilon_{m}-\epsilon_{0} \simeq s^{\prime} \hbar 2 \pi / \tau$. As mentioned above, we also have $\epsilon_{m}-\epsilon_{0} \simeq m \hbar \omega_{0}+s^{\prime \prime} 2 \pi / \tau$ from a semiclassical argument ( $s^{\prime \prime}$ being an integer). Hence, we immediately see that $m$ must be an integer multiple of $\ell$.

The analysis given so far, however, shows that the tunnelling enhancement cannot be explained with just one crossing of the $\epsilon_{0}^{ \pm}$doublet by $\epsilon_{m}^{ \pm}$. This global crossing is actually made up of many elementary crossings whose contributions cannot be individually distinguished. In effect, this game involves many players among which are the states that are completely delocalised in the chaotic sea. There are also states ("beach" states [41, 42] or "Janus" states) that are strongly coupled on one side to the regular states and on the other side to the chaotic ones. It is scarcely surprising that these states have their Husimi distribution localised near the borderline between the stable islands and the chaotic sea (see FIG. 5).

\section{RESONANCE-ASSISTED TUNNELLING}

The above discussion has provided overwhelming evidence for the relevance of nonlinear resonances in the dynamical tunnelling process. We now focus on the quantitative evaluation of the influence of such resonances, which was presented in detail in Refs. 24, 25, 26, 27]. To this end, we formally introduce an integrable Hamiltonian that approximately reproduces the dynamics within the regular island. For the upper island at $p \simeq 1$, such an integrable system can be explicitly obtained by leaving out the $\gamma_{+}$-dependent term in the Hamiltonian (1). Performing the time-dependent canonical transformation $q \mapsto \tilde{q}=q-t$ to the frame that co-propagates with the resonant orbit, this integrable Hamiltonian reads

$$
H_{0}(p, \tilde{q})=\frac{(p-1)^{2}}{2}-\frac{\gamma_{-}}{2} \cos (\tilde{q})
$$

Canonical perturbation theory 31] can be applied on the basis of Eq. (3) in order to obtain an improved integrable description that is in good agreement with the motion in the regular island also at finite values of $\gamma$.

In the dynamics generated by $H_{0}$, the regular island is embedded in a phase-space domain of bounded elliptic motion in momentum space. Within this bounded domain, action-angle variables $(I, \theta)$ can be introduced, which respectively correspond to the area enclosed by an elliptic invariant orbit as well as to the propagation time that elapses along this orbit. In this action-angle variable representation, we have $H_{0}(p, \tilde{q}) \equiv H_{0}(I)$, and the full time-dependent Hamiltonian (11) is formally written
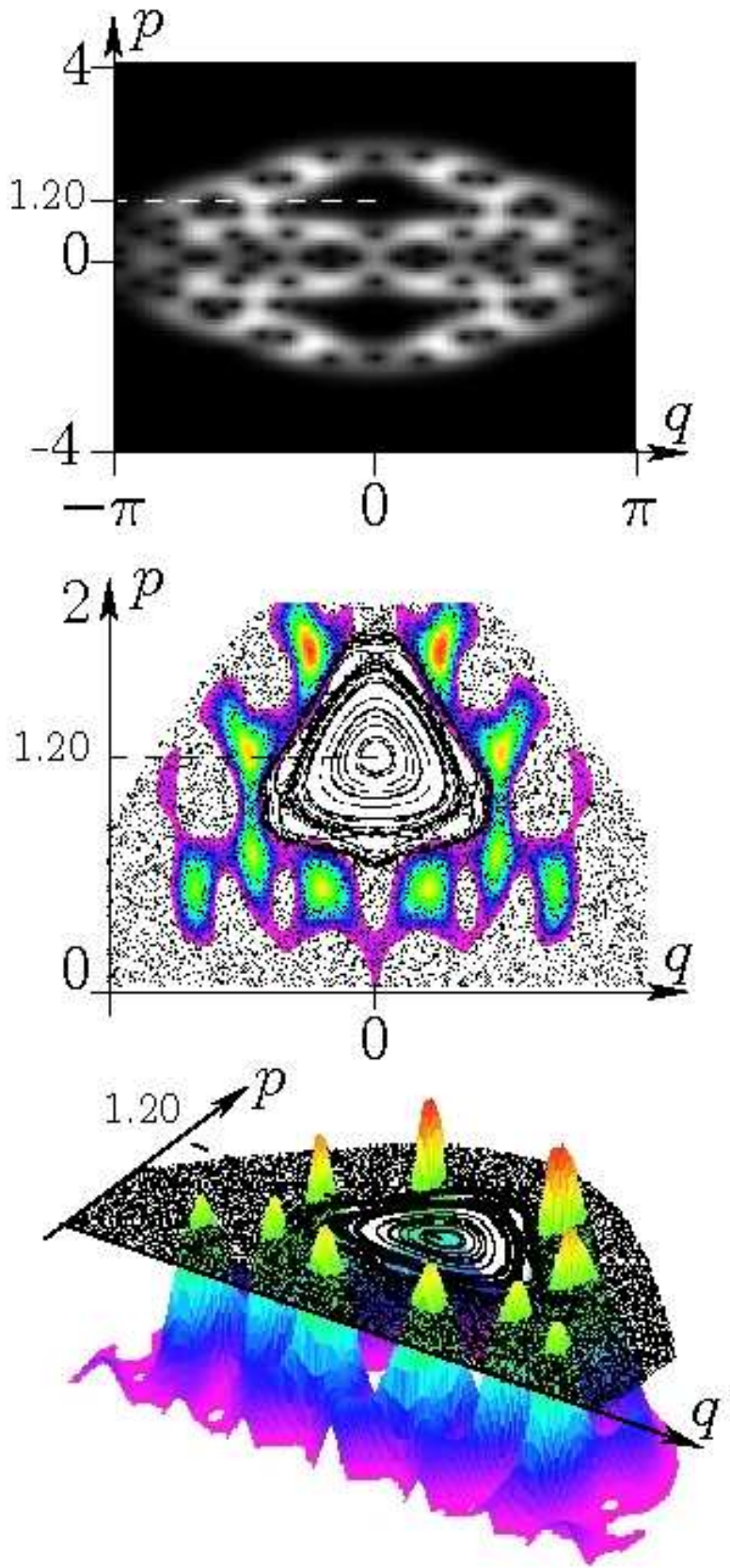

FIG. 5: A Janus state: it is strongly coupled to the most excited resonant state in the stable island and to the chaotic states. In the random matrix model, it is "seen" as chaotic from the regular states and almost "regular" from the chaotic sea. Here we plot several views of the Husimi distribution of a Janus state and compare its localisation with the classical phase-space structures $\left(\gamma=0.72, \hbar^{-1}=30\right.$, and $\epsilon=-0.007774)$.

as $H(I, \theta, t)=H_{0}(I)+V(I, \theta, t)$ where $V$ represents a weak perturbation within the island.

We now assume the presence of a prominent $s / \ell$ resonance within the regular island, where $s$ oscillations around the island's center match $\ell$ periods of the driv- 


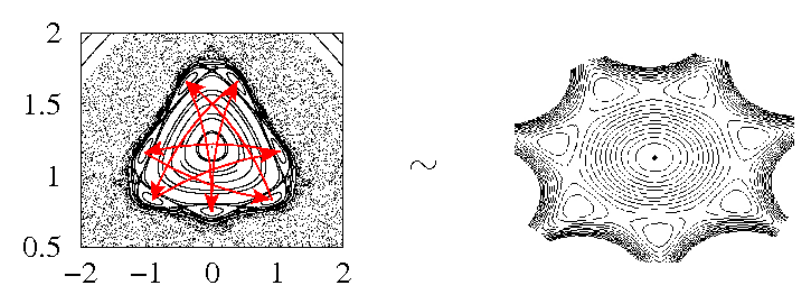

FIG. 6: Left panel: classical phase space in the vicinity of the regular island. The straight solid lines indicate in which order the sub-islands are "visited" in the course of time evolution. The right panel shows the phase space structure that would result from the pendulum-like Hamiltonian (4) describing the dynamics in the vicinity of the $3 / 7$ resonance.

ing. This resonance condition is satisfied at $\ell \omega_{0}(I)=s$ where $\omega_{0} \equiv d H_{0} / d I$ is the oscillation frequency along the bounded orbit with action variable $I$. The dynamics in the vicinity of such a resonance can be approximately described by the pendulum-like integrable Hamiltonian

$$
H_{\mathrm{eff}}(I, \vartheta)=\frac{\left(I-I_{0}\right)^{2}}{2 m_{0}}+2 V_{0} \cos (\ell \vartheta)
$$

which is derived from $H(I, \theta, t)$ using secular perturbation theory [31]. Here, $\vartheta=\theta-(s / \ell) t$ is the (slowly varying) angle variable that co-rotates with the resonance. The resulting phase space structure of $H_{\mathrm{eff}}$ is plotted in Fig. [6.

Following the lines of Ref. [43], we now evaluate the influence of such a resonance in the corresponding quantum system by a direct quantization of the effective pendulum Hamiltonian (44) in the modified angle variable $\vartheta$. Apart from a phase factor containing the Maslov index, the unperturbed eigenstates of $H_{0}$ are then given by the plane waves $\langle\vartheta \mid n\rangle=\exp (i n \vartheta)$. In the co-rotating frame, their eigenenergies approximately read

$$
E_{n}=\frac{\left[\hbar(n+1 / 2)-I_{0}\right]^{2}}{2 m_{0}}
$$

using the fact that $I_{n}=\hbar(n+1 / 2)$ are the quantized action variables within a regular island of elliptic shape. The $\vartheta$-dependent term in Eq. (44) introduces couplings between the states $|n\rangle$ and $|n \pm \ell\rangle$ with the coupling matrix element $V_{0}$. These couplings give rise to perturbative chains by means of which eigenstates with low and high excitations within the bounded domain are connected to each other.

The pendulum Hamiltonian (4) can be considered to be appropriate for $I<I_{c}$ where $I_{c}$ denotes the action variable of the outermost invariant elliptic curve of the regular island. The regime beyond this "chaos border" is characterized by the presence of multiple overlapping resonances, which implies that the unperturbed states with $I_{n}>I_{c}$ can be assumed to be strongly coupled to each other by many different matrix elements. Such couplings would also occur between "bound" and "unbound" eigenstates of the integrable Hamiltonian $H_{0}$ (which are, respectively, located within and outside the domain of bounded motion that embeds the regular island) as well as between states that are located in the vicinity of the two different islands at $p \simeq 1$ and $p \simeq-1$. In this way, an efficient two-step mechanism is introduced by which two symmetry-related quasimodes that are localized in the upper and lower island, respectively, are coupled to each other: the nonlinear resonance connects those quasimodes to the states in the chaotic domain, and the latter "see" each other via strong matrix elements of the full (Floquet) Hamiltonian of the driven system.

Fig. [7 schematically displays the effective Hamiltonian matrix that governs this resonance- and chaos-assisted tunnelling process between the two "central states" of the islands (i.e., given by $|n=0\rangle$ in the above notation). The matrix is restricted to basis states of one particular symmetry class - i.e., to states that are "even" or "odd" with respect to time-reversal symmetry - and includes, for the sake of clarity, only those regular components to which the central state is perturbatively connected via the $s / \ell$ resonance. Altogether, $k_{c}$ regular states are included in the matrix, where the integer $k_{c}$ is defined such that $I_{\left(k_{c}-1\right) \ell}<I_{c}<I_{k_{c} \ell}$ holds true, i.e., $\left|\left(k_{c}-1\right) \ell\right\rangle$ is still located in the island, whereas $\left|k_{c} \ell\right\rangle$ is dissolved in the chaotic part.

We can now prediagonalize the upper left "regular" block of the Hamiltonian. This yields, in lowest nonvanishing order in the perturbation strength $\left|V_{0}\right|$ (which is much smaller than all relevant energy differences), the modified central state as

$$
|\tilde{0}\rangle=|0\rangle+\sum_{k=1}^{k_{c}-1}\left(\prod_{k^{\prime}=1}^{k} \frac{V_{0}}{E_{0}-E_{k^{\prime} \ell}}\right)|k \ell\rangle .
$$

This perturbed central now exhibits a nonvanishing matrix element with one of the states that are contained within the chaotic domain: we obtain

$$
V_{\text {eff }} \equiv\left\langle k_{c} \ell|H| \tilde{0}\right\rangle=V_{0} \prod_{k=1}^{k_{c}-1} \frac{V_{0}}{E_{0}-E_{k \ell}}
$$

which can be interpreted as the effective resonanceinduced matrix element between the central state and the chaotic domain. In this way, our effective Hamiltonian matrix can be related to the phenomenological matrix models that constitute the starting point of the statistical theory of chaos-assisted tunnelling [21, 22, 23].

In the simplest possible approach, we assume that the "chaos block" is essentially homogeneous and can be well modeled by a random matrix from the Gaussian Orthogonal Ensemble (GOE). This assumption does not account for the influence of prominent partial barriers to the classical transport, which can arise from broken invariant tori (so-called "Cantori" [4]) as well as from unstable periodic orbits in the chaotic sea, and which would lead to an effective division of the chaotic Hamiltonian into several sub-blocks that are weakly connected to each other [21]. 


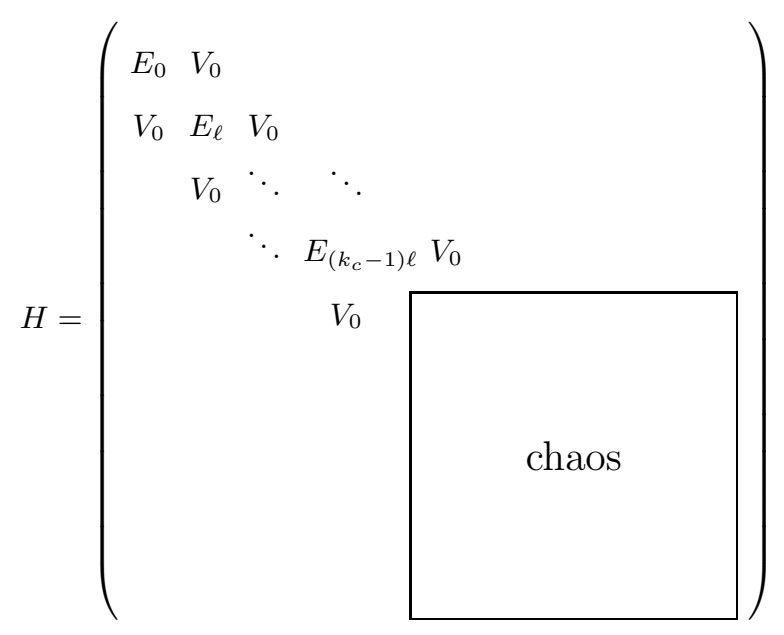

FIG. 7: Sketch of the effective Hamiltonian matrix that describes the coupling between the regular island and the chaotic domain for one particular symmetry class (i.e., for "even" or "odd" states with respect to time-reversal symmetry). The regular part (upper left band) includes only components that are coupled to the island's central state by the $s / \ell$ resonance. In the simplest possible approximation, the chaotic part consists of a full sub-block with equally strong couplings between all basis states with actions beyond the outermost invariant torus of the island.

Neglecting those partial barriers and performing the random matrix average over the eigenvectors and eigenvalues of the chaos block gives rise to the Cauchy distribution

$$
P\left(\Delta E_{0}\right)=\frac{2}{\pi} \frac{\overline{\Delta E_{0}}}{\left(\Delta E_{0}\right)^{2}+\left(\overline{\Delta E_{0}}\right)^{2}}
$$

for the level splitting between the "even" and "odd" combination of central states that are associated with the pair of regular islands 22, 23]. This distribution is characterized by the scale

$$
\overline{\Delta E_{0}}=\frac{2 \pi V_{\mathrm{eff}}^{2}}{N_{c} \Delta_{c}}
$$

which contains the most dominant effective matrix element (7) between the island's central state and one of the chaotic states, the total number $N_{c}$ of chaotic states, as well as the mean level spacing $\Delta_{c}$ in the chaos block. In our case of a periodically driven system, the latter is given by $\Delta_{c}=\hbar / N_{c}$, due to the fact that the chaotic states are, in the framework of the Floquet approach, uniformly distributed at random in an energy window of the size $2 \pi \hbar / \tau=\hbar$ (we recall that the period of the driving equals $\tau=2 \pi$ ). As a consequence, we obtain $\overline{\Delta E_{0}}=2 \pi V_{\text {eff }}^{2} / \hbar$.

The distribution (8) is, strictly speaking, valid only for $\Delta E_{0} \ll V_{\text {eff }}$ and exhibits a cutoff at $\Delta E_{0} \sim 2 V_{\text {eff }}$ 23], which ensures that the statistical expectation value $\left\langle\Delta E_{0}\right\rangle=\int_{0}^{\infty} x P(x) d x$ does not diverge. However, since tunnelling rates and their parametric variations are typically studied on a logarithmic scale (see Fig. 2), we compute from Eq. (8) not the mean value $\left\langle\Delta E_{0}\right\rangle$, but rather the average of the logarithm of $\Delta E_{0}$. Our "average" level splitting $\left\langle\Delta E_{0}\right\rangle_{g}$ is therefore defined by the geometric mean $\left\langle\Delta E_{0}\right\rangle_{g} \equiv \exp \left[\left\langle\ln \left(\Delta E_{0}\right)\right\rangle\right]$ the evaluation of which does not involve the above cutoff; we obtain the expression

$$
\left\langle\Delta E_{0}\right\rangle_{g}=\overline{\Delta E_{0}}=\frac{2 \pi V_{\mathrm{eff}}^{2}}{\hbar}
$$

which, notably, is free of any adjustable parameter. Hence, up to a trivial prefactor, the mean value of splittings is, in a logarithmic-scale representation, given by the square of the coupling matrix element (7) between the island's central state and the chaos [26, 27].

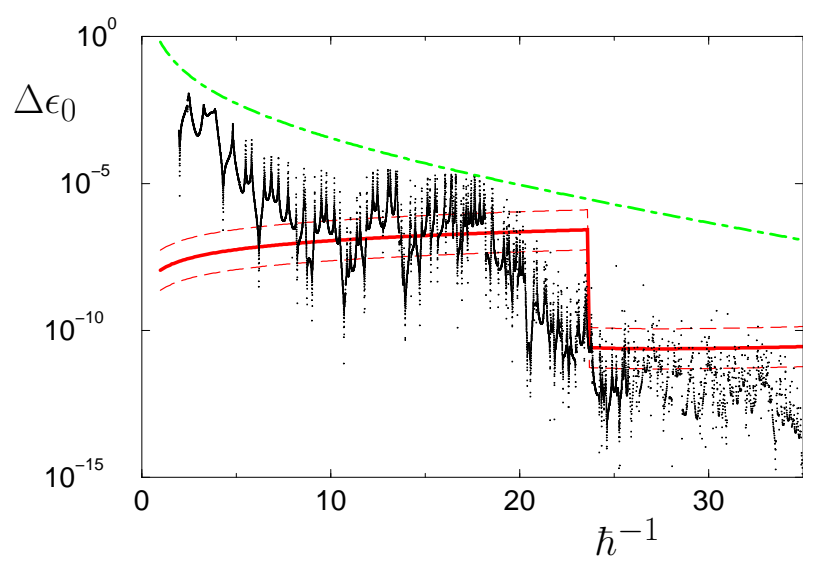

FIG. 8: Comparison between the numerically calculated splittings of the driven pendulum at $\gamma=0.72$ (black dots) and the semiclassical prediction according to Eq. 10 (red solid line). The semiclassical theory, which is based on the prominent internal $3 / 7$ resonance within the regular island, reproduces quite well the positions and heights of the two plateaus that appear in the exact quantum splittings. The dashed lines indicate the size of the logarithmic standard deviation according to Eq. (11). The green dot-dashed line displays the prediction that would be obtained from Eq. (12) with $\Omega=1$.

Figure 8 shows the comparison with the exact quantum splittings at $\gamma=0.72$, calculated by the numerical diagonalization of the Floquet matrix. The semiclassical prediction (10) (solid line in Fig. 8) was evaluated on the basis of the prominent $3 / 7$ resonance, for which the relevant parameters $I_{0}, m_{0}$ and $V_{0}$ that enter into the pendulum Hamiltonian (4) were entirely determined from classical dynamics of the system: As in Ref. [26], we compute for this purpose the trace of the monodromy matrix associated with a stable or unstable periodic point of the $3 / 7$ resonance, as well as the phase space areas that are enclosed by the inner and outer separatrices of the resonance. Indeed, those quantities remain invariant 
under the canonical transformation to the action-angle variables $(I, \vartheta)$, which means that the latter need not be explicitly evaluated in order to obtain the effective coupling matrix element $V_{\text {eff }}$.

We see that the semiclassical theory reproduces quite well the two plateaus that arise in the quantum splittings. The drop in the semiclassical splittings at $1 / \hbar \simeq 24$ occurs due to the fact that the island supports more than seven locally quantized eigenstates beyond this critical value of $1 / \hbar$; hence, two perturbative steps instead of one are required in order to connect the central state of the island to the chaotic domain. This drop is considerably softened in the quantum splittings, but can still be identified, which confirms the relevance of the resonanceassisted coupling mechanism in this tunnelling process. Since nonlinear resonances represent a general feature of nonintegrable Hamiltonian systems, we expect that the appearance of such step-like sequences of plateaus is a generic phenomenon, which is not restricted to one particular system, but arises in various chaos-assisted tunnelling processes. This expectation is indeed confirmed by previous studies on the kicked Harper [40], on the decay of nondispersive wave packets in driven hydrogen [45], as well as on the dynamical tunnelling process of cold atoms [3, 46], where significant plateau structures were encountered in numerically calculated tunnelling rates.

In addition to the mean value for the splittings, the probability distribution (8) can also be used in order to calculate the expectation value for their logarithmic variance characterizing the average size of fluctuations on a logarithmic scale; we obtain

$$
\left\langle\left(\ln \Delta E_{0}-\ln \overline{\Delta E_{0}}\right)^{2}\right\rangle=\pi^{2} / 4 .
$$

This result is universal in the sense that it does not depend on system-specific parameters nor on $\hbar$. The size of the corresponding standard deviation is indicated by the dashed lines in Fig. 8 which are generated by multiplying $\overline{\Delta E_{0}}$ with $e^{ \pm \pi / 2}$. Clearly, the "window" defined by those dashed lines is exceeded near local avoided crossings with chaotic states, where a large enhancement as well as a complete suppression of the splittings may be induced [2]. Apart from those exceptional events, however, the scale of the average fluctuations of the splittings is well described by Eq. (11). The green dot-dashed line in Fig. 8 displays the prediction for the splittings that would be obtained from Eq. (2) according to Ref. [28]. Here, the asymptotic expression

$$
\Delta \epsilon \simeq \frac{\hbar \Omega}{16 \pi N^{3}} \mathrm{e}^{-2(1-\ln 2) N}
$$

of Eq. (2), valid for $N \equiv A /(2 \pi \hbar) \gg 1$, was evaluated with the numerically calculated size $A$ of the island. Being an entirely classical ( $\hbar$-independent) quantity, the system-dependent prefactor $\Omega$ was set equal to unity. We clearly note a substantial disagreement between this semiclassical estimation and the quantum splittings.
The theory of resonance-assisted tunnelling does not reproduce the splittings in the deep quantum regime of larges values of $\hbar$, where a different mechanism, possibly in the spirit of Ref. [28], might induce the transition to the chaos. Moreover, the critical value of $1 / \hbar$ at which the drop of the splittings from the first to the second plateau occurs is significantly overestimated by our semiclassical approach. This could be due to the presence of partial barriers in the chaotic phase space domain, such as "Cantori" [4], which are known to inhibit the quantum flux at not too small values for $\hbar$ [47, 48]. If such a Cantorus is manifested in the vicinity of the regular island, the effective "quantum" size of the island could be considerably enhanced as compared to $A$, which would reduce the value of $1 / \hbar$ at which exactly $\ell$ quantum states are localized around the island. This observation is indeed in accordance with the manifestation of Janus states 41, 42] in the spectral analysis (see Section IV).

Significant deviations of the splittings from the semiclassical prediction (10) are also to be expected in the deep semiclassical regime where a multitude of steps $(k \gg 1)$ would be needed to connect the central state to the chaotic domain according to the expression (7). In this regime, the coupling via the $s / \ell$ resonance which also represents a dynamical tunnelling process as was pointed out in Ref. 25] — can again be assisted by the presence of another nonlinear $s^{\prime} / \ell^{\prime}$ resonance, as long as this is permitted by the associated selection rule $\left(n \mapsto n+\ell^{\prime}\right)$. Such a $s^{\prime} / \ell^{\prime}$ resonance would generally exhibit a lower effective coupling strength $V_{0}^{\prime}$ and is typically of higher order than the $s / \ell$ resonance (i.e., $\ell^{\prime}$ and $s^{\prime}$ are typically larger than $\ell$ and $s$, respectively). The relevance of this multi-resonance coupling mechanism was demonstrated in the near-integrable kicked Harper model where the semiclassical tunnelling process involves a sequence of three nonlinear resonances [24, 25, 27].

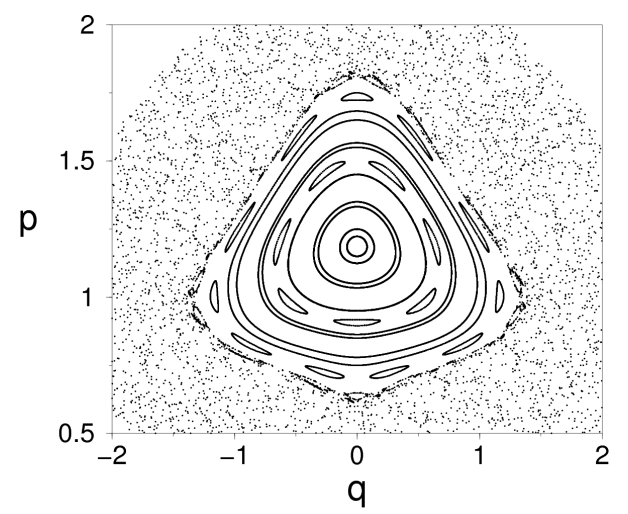

FIG. 9: Classical phase space at $\gamma=0.67$ in the vicinity of the regular island $\mathcal{I}_{+}$. The two chains of sub-islands correspond to the $3 / 7$ as well as to the $5 / 11$ resonance (inner and outer chain, respectively).

In the case of the driven pendulum at $\gamma=0.72$, such multi-resonance processes cannot be observed within the 
range of values for $1 / \hbar$ at which precise quantum calculations of the splittings can be performed. This is different, however, for $\gamma=0.67$. At this value of the coupling parameter, the regular island $\mathcal{I}_{ \pm}$exhibits two nonlinear resonances of almost equal importance: a $3 / 7$ resonance, located closer to the center of the island than at $\gamma=0.72$, and a 5/11 resonance, located close to the chaos border (see Fig. 9). This implies that a two-step tunnelling process involving both resonances can be encountered at finite values for $1 / \hbar$. Assuming that each resonance contributes with one single perturbative step (which is the case at the values of $1 / \hbar$ considered here), the corresponding matrix element that connects the central state to the chaotic domain would read

$$
V_{\mathrm{eff}}=\frac{V_{0}^{(3 / 7)}}{E_{0}^{(3 / 7)}-E_{7}^{(3 / 7)}} V_{0}^{(5 / 11)}
$$

Here, $V_{0}^{(s / \ell)}$ represents the pendulum coupling strength of the effective Hamiltonian (4), and $E_{n}^{(s / \ell)}$ denote the energies (5) in the co-rotating frame that is defined with respect to the $s / \ell$ resonance. This expression requires that the 7 th excited state, i.e. the first state to which the central state of the island is coupled via the $3 / 7$ resonance, is located in between the two resonances, which implies that the action variable $I_{7}$ of this state ought to be lower than the action variable $I_{0}^{(5 / 11)}$ of the $5 / 11$ resonance. This condition turns out to be valid for $1 / \hbar>28.8$.

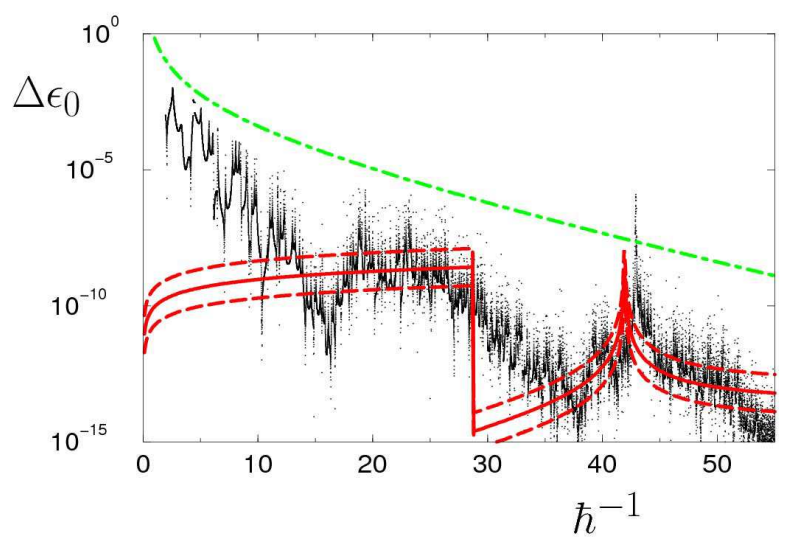

FIG. 10: Comparison between the numerically calculated splittings of the driven pendulum at $\gamma=0.67$ (black dots) and the semiclassical prediction (red solid line). The latter was evaluated by a single-resonance process via the $5 / 11$ resonance for $1 / \hbar<28.8$, and by a two-resonance process according to Eq. 13 involving also the $3 / 7$ resonance for $1 / \hbar>28.8$. As in Fig. 8 the dashed lines indicate the size of the logarithmic standard deviation according to Eq. (11), and the green dotdashed line displays the prediction obtained from Eq. (12).

Fig. 10] displays the quantum splittings at $\gamma=0.67$. The red solid line denotes the semiclassical prediction (10) for the splittings, which is for $1 / \hbar<28.8$ calculated by the single-step process via the $5 / 11$ resonance (which has the larger matrix element $V_{0}^{(s / \ell)}$ and should therefore dominate compared to the $3 / 7$ resonance) and for $1 / \hbar>28.8$ obtained through the two-step process that is described by Eq. (13). As in the case of $\gamma=0.72$, the overall agreement between the semiclassical and the quantum splittings is quite good, with significant deviations arising only in the deep quantum regime at $1 / \hbar<10$ as well as in the vicinity of the cross-over between the single- and the two-step process, which is artificially sharp in the semiclassical calculation. Striking evidence for the validity of the two-step process is the appearance of a pronounced peak in the quantum splittings at $1 / \hbar \simeq 43$, which arises due to a vanishing denominator in Eq. (13), i.e., due to the fact the central state and the 7 th excited state become near-degenerate in the corotating frame. The position and height of this peak are fairly well reproduced by the semiclassical theory.

\section{CONCLUSION}

In summary, we provided clear evidence of the significance of nonlinear resonances in the dynamical tunnelling process within the driven pendulum. Indeed, the signature of the $3 / 7$ resonance that is dominantly manifested in the regular island at $\gamma=0.72$ is identified in various ways: (a) The characteristic plateau structure in the level splittings sensitively depends on whether or not the resonance is well developed within the regular island, (b) Floquet states that exhibit an appreciable overlap with the central Husimi wavefunction of the island energetically correspond to the 7th excited eigenstate within the island, and (c) a semiclassical expression for the tunnelling-induced splittings that is based on the resonance-assisted coupling scheme is in good agreement with the exact quantum data. The validity of the resonance-assisted tunnelling mechanism is furthermore confirmed by a striking peak in the quantum splittings at $\gamma=0.67$, which arises due to a two-step process involving two different nonlinear resonances within the regular island. Both in the spectral analysis and in the semiclassical comparison, we identify traces of Janus states that are located in the chaotic vicinity of the islands. This indicates that partial barriers in the chaotic domain could still be relevant in our system at the values of $\hbar$ under consideration.

The present approach is presumably not suited for a full-blown semiclassical theory of mixed regular-chaotic tunnelling in terms of complexified orbits, for which the ansatz of Refs. 16, 17, 18] provides a more convenient framework. Our findings, however, provides essential ingredients to the interpretation of such semiclassical theories, in the sense that "direct" and "resonance-assisted" processes ought to be somehow represented in relevant combinations of such complex orbits. Moreover, specific quantitative predictions for tunnelling rates, using only easily accessible quantities of the classical dynamics of the system, can be made on the basis of our approach, 
provided $\hbar$ is small enough for the most dominant resonance to become relevant (which is roughly the case if $\ell / 2$ states fit into the island). We therefore believe that the principle of resonance-assisted couplings will represent the relevant paradigm also in the context of more complex dynamical tunnelling processes, e.g., within systems that have two or more degrees of freedom.

Acknowledgments: It is a pleasure to thank Denis
Ullmo for sharing with us his extensive experience on tunnelling. A.M. acknowledges the generous hospitality of Dominique Delande at Laboratoire Kastler-Brossel and his relevant remarks after reading the first proof of this manuscript. P.S. acknowledges support from the Bayerisch-Französisches Hochschulzentrum (BFHZ) and from the Deutsche Forschungsgemeinschaft (DFG).
[1] W. A. Lin and L. E. Ballentine, Phys. Rev. Lett. 65, 2927 (1990).

[2] F. Grossmann, T. Dittrich, P. Jung, and P. Hänggi, Phys. Rev. Lett. 67, 516 (1991).

[3] A. Mouchet, C. Miniatura, R. Kaiser, B. Grémaud, and D. Delande, Phys. Rev. E 64, 016221 (2001).

[4] W. K. Hensinger, H. Häffner, A. Browaeys, N. R. Heckenberg, K. Helmerson, C. McKenzie, G. J Milburn, W. D Phillips, S. L. Rolston, H. Rubinsztein-Dunlop and B. Upcroft, Nature 412, 52 (2001).

[5] D. A. Steck, H. O. Windell, and M. G. Raizen, Science 293, 274 (2001).

[6] W. K. Hensinger, A. Mouchet, P. S. Julienne, D. Delande, N. R. Heckenberg and H. Rubinsztein-Dunlop, Phys. Rev. A (3) 70, 013408 (2004).

[7] C. Dembowski, H.-D. Gräf, A. Heine, R. Hofferbert, H. Rehfeld and A. Richter, Phys. Rev. Lett. 84, 867 (2000).

[8] R. Hofferbert, H. Alt, C. Dembowski, H.-D. Gräf, H. L. Harney, A. Heine, H. Rehfeld and A. Richter, Phys. Rev. E 71, 046201 (2005).

[9] L. D. Landau and E. M. Lifshitz, Quantum Mechanics (non relativistic theory), Vol. 3 of Course of Theoretical Physics (Pergamon Press, Oxford, 1958).

[10] R. Balian and C. Bloch, Ann. Physics 84, 559 (1974).

[11] M. Kuś, F. Haake, and D. Delande, Phys. Rev. Lett. 71, 2167 (1993).

[12] P. Leboeuf and A. Mouchet, Phys. Rev. Lett. 73, 1360 (1994).

[13] R. Scharf and B. Sundaram, Phys. Rev. E 49, R4767 (1994).

[14] S. C. Creagh and N. D. Whelan, Phys. Rev. Lett. 77, 4975 (1996).

[15] For multidimensional non-separable but integrable systems, these tori are precisely the relevant classical structures that allow to generalise JWKB 1d-tunnelling [38].

[16] A. Shudo and K. S. Ikeda, Phys. Rev. Lett. 74, 682 (1995).

[17] A. Shudo and K. S. Ikeda, Physica D 115, 234 (1998).

[18] A. Shudo, Y. Ishii, and K. S. Ikeda, J. Phys. A 35, L225 (2002).

[19] O. Bohigas, M.-J. Giannoni, and C. Schmit, Phys. Rev. Lett. 52, 1 (1983).

[20] O. Bohigas, D. Boosé, R. E. de Carvalho, and V. Marvulle, Nuclear Phys. A 560, 197 (1993).

[21] O. Bohigas, S. Tomsovic, and D. Ullmo, Phys. Rep. 223, 43 (1993).

[22] S. Tomsovic and D. Ullmo, Phys. Rev. E 50, 145 (1994).

[23] F. Leyvraz and D. Ullmo, J. Phys. A 29, 2529 (1996).

[24] O. Brodier, P. Schlagheck, and D. Ullmo, Phys. Rev. Lett. 87, 064101 (2001).

[25] O. Brodier, P. Schlagheck, and D. Ullmo, Ann. Physics
300, 88 (2002).

[26] C. Eltschka and P. Schlagheck, Phys. Rev. Lett. 94, 014101 (2005).

[27] P. Schlagheck, C. Eltschka, and D. Ullmo, in Progress in Ultrafast Intense Laser Science I, edited by K. Yamanouchi, S. L. Chin, P. Agostini, and G. Ferrante (Springer, Berlin, 2006), p. 107.

[28] V. A. Podolskiy and E. E. Narimanov, Phys. Rev. Lett. 91, 263601 (2003).

[29] A. Mouchet and D. Delande, Phys. Rev. E 67, 046216 (2003).

[30] B. Chirikov, Phys. Rep. 52, 263 (1979).

[31] A. J. Lichtenberg and M. A. Lieberman, Regular and Stochastic Motion, Vol. 38 of Applied Mathematical Sciences (Springer-Verlag, New York, 1983).

[32] The only difference is that a third resonance term must be added to (11) in order to fulfil some experimental constraints on the lasers configuration. How this can be done is explained in Refs. [3, 4, 5] but the present paper, devoted to theoretical considerations only, will not introduce this irrelevant third term.

[33] M. J. Davis and E. J. Heller, J. Phys. Chem. 85, 307 (1981).

[34] M. Latka, P. Grigolini, and B. J. West, Phys. Rev. E 50, 596 (1994).

[35] V. Averbukh, N. Moiseyev, B. Mirbach, and H. J. Korsh, Z. Phys. D 35, 247 (1995).

[36] L. Bonci, A. Farusi, P. Grigolini, and R. Roncaglia, Phys. Rev. E 58, 5689 (1998).

[37] For the same reason we will not consider the different possible boundary conditions offered by Bloch's theorem. The wave-functions in the $q$ representation will always be taken to be strictly $2 \pi$ spatially periodic, i.e. without acquiring any phase-factor when winding around the cylinder.

[38] S. C. Creagh, J. Phys. A 27, 4969 (1994).

[39] R. Egydio de Carvalho and A. P. Mijolaro, Phys. Rev. E 70, 056212 (2004).

[40] R. Roncaglia, L. Bonci, F. M Izrailev, B. J. West, and P. Grigolini, Phys. Rev. Lett. 73, 802 (1994).

[41] E. Doron and S. D. Frischat, Phys. Rev. Lett. 75, 3661 (1995).

[42] S. D. Frischat and E. Doron, Phys. Rev. E 57, 1421 (1998).

[43] A. M. Ozorio de Almeida, J. Phys. Chem. 88, 6139 (1984).

[44] R. S. MacKay, J. D. Meiss, and I. C. Percival, Phys. Rev. Lett. 52, 697 (1984).

[45] K. Hornberger and A. Buchleitner, Europhys. Lett. 41, 383 (1998).

[46] For this latter case, we have indications that the promi- 
nent plateau structure in the level splittings at $\gamma=0.18$ (see Fig. 7 in Ref. [3]) is induced by a nonlinear $1 / 4$ resonance that is already contained within the chaotic part of the phase space. Since our numerical approach to calculate the coupling matrix element $V_{0}$ is perturbative by nature and relies on a well-preserved separatrix structure of the resonance, we are, at present, unable to make a quantitative semiclassical reproduction of this particular plateau.

[47] T. Geisel, G. Radons, and J. Rubner, Phys. Rev. Lett. 57, 2883 (1986).

[48] N. T. Maitra and E. J. Heller, Phys. Rev. E 61, 3620 (2000). 